\title{
Spatial and radiometric characterization of multi-spectrum satellite images through multi-fractal analysis
}

\author{
Carmelo Alonso $^{1,2}$, Ana M. Tarquis ${ }^{2,3}$, Ignacio Zúñiga ${ }^{4}$, and Rosa M. Benito ${ }^{2}$ \\ ${ }^{1}$ Earth Observation Systems, Indra Sistemas S.A., Madrid, Spain \\ ${ }^{2}$ Grupo de Sistemas Complejos, U.P.M, Madrid, Spain \\ ${ }^{3}$ CEIGRAM, E.T.S.I.A.A.B., U.P.M, Madrid, Spain \\ ${ }^{4}$ Dpt. Física Fundamental, Facultad de Ciencias, Universidad Nacional de Educación a Distancia (UNED), Madrid, Spain \\ Correspondence to: Ana M. Tarquis (anamaria.tarquis@upm.es)
}

Received: 20 May 2016 - Discussion started: 5 August 2016

Revised: 3 February 2017 - Accepted: 22 February 2017 - Published: 16 March 2017

\begin{abstract}
Several studies have shown that vegetation indexes can be used to estimate root zone soil moisture. Earth surface images, obtained by high-resolution satellites, presently give a lot of information on these indexes, based on the data of several wavelengths. Because of the potential capacity for systematic observations at various scales, remote sensing technology extends the possible data archives from the present time to several decades back. Because of this advantage, enormous efforts have been made by researchers and application specialists to delineate vegetation indexes from local scale to global scale by applying remote sensing imagery.

In this work, four band images have been considered, which are involved in these vegetation indexes, and were taken by satellites Ikonos- 2 and Landsat- 7 of the same geographic location, to study the effect of both spatial (pixel size) and radiometric (number of bits coding the image) resolution on these wavelength bands as well as two vegetation indexes: the Normalized Difference Vegetation Index (NDVI) and the Enhanced Vegetation Index (EVI).

In order to do so, a multi-fractal analysis of these multispectral images was applied in each of these bands and the two indexes derived. The results showed that spatial resolution has a similar scaling effect in the four bands, but radiometric resolution has a larger influence in blue and green bands than in red and near-infrared bands. The NDVI showed a higher sensitivity to the radiometric resolution than EVI. Both were equally affected by the spatial resolution.

From both factors, the spatial resolution has a major impact in the multi-fractal spectrum for all the bands and the
\end{abstract}

vegetation indexes. This information should be taken in to account when vegetation indexes based on different satellite sensors are obtained.

\section{Introduction}

Soil moisture is a critical condition affecting interaction of land surface and atmosphere. Remotely sensed data are an important source of information and can indirectly measure soil moisture in space and time. However, the signal only penetrates the top few centimetres, and soil moisture at deeper layers must be estimated. One method to estimate soil moisture at deeper layers is through vegetation indexes. Several authors have investigated the potential of vegetation indexes to estimate root zone soil moisture. The Normalized Difference Vegetation Index (NDVI) and Enhanced Vegetation Index (EVI) have been used by several authors (Wang et al., 2007; Ben-Ze'ev et al., 2006; Deng et al., 2007) in different conditions to find significant estimations with root zone soil moisture. For the estimation of these indexes nearinfrared (NIR), red and blue wavelengths are needed (Huete et al., 2014).

The images provided by the satellites show the land surface in a wide range of wavelengths (from visible to thermal infrared or microwaves) and also with a great variety of spatial resolutions (from a few kilometres to tens of centimetres). The analysis of these varied images and their synergic possibilities are a challenging problem, especially with new sensors, which have small spatial resolution and a large range 
of radiometric quantification. Fractal analysis offers significant potential for improvement in the measurement and analysis of spatially and radiometrically complex remote sensing data. This analysis also provides quantitative insight on the spatial complexity in the information of the landscape contained within these data.

In the general mathematical framework of fractal geometry, many analytical methods have been developed, including the following: textural homogeneity, which has been characterized using the fractal dimension (Fioravanti, 1994) (it has also been used as a spatial measure for describing the complexity of remote sensing imagery; Lam and De Cola, 1993)); and changes in the image complexity, which have been detected through the spectral range of hyperspectral images affecting the fractal dimension (Qiu et al., 1999). Similarly, De Cola (1989) and Lam (1990) have found that fractal dimension also depends on the spectral bands of Landsat-7 TM imagery.

Motivated by the fractal geometry of sets (Mandelbrot, 1983), the development of multi-fractal theory, introduced in the context of turbulence, has been applied in many areas, such as earthquake distribution analysis (Hirata and Imoto, 1991), soil pore characterization (Kravchenko et al., 1999; Tarquis et al., 2003), image analysis (Sánchez et al., 1992) or remote sensing (Tessier et al., 1993; Cheng and Agterberg, 1996; Schmitt et al., 1997; Laferrière and Gaonac'h, 1999; Cheng, 2004; Lovejoy et al., 2001b; Du and Yeo, 2002; Parrinello and Vaughan, 2002; Harvey et al., 2002; Turiel et al., 2005).

The acquisition of remotely sensed multiple spectral images is thus a unique source of data for determining the scaleinvariant characteristics of the radiance fields related to many factors, such as soil and bedrock chemical composition, humidity content and surface temperature (e.g. Laferrière and Gaonac'h, 1999; Maìtre and Pinciroli, 1999; Lovejoy et al., 2001a, b; Harvey et al., 2002; Beaulieu and Gaonac'h, 2002; Gaonac'h et al., 2003). In one of the schemes used in the multi-fractal analysis, the satellite image is considered as a mass distribution of a statistical measure on the space domain studied, and it is analysed through a multi-fractal (MF) spectrum (Cheng, 2004; Mao-Gui Hu, 2009; Tarquis et al., 2014), which gives either geometrical or probabilistic information about the pixel distribution with the same singularity. Other techniques focus on the variations of a measure analysing the moments of the absolute differences of their values at different scales, e.g. the Generalized Structure Function and the Universal Multi-fractal model (Lovejoy et al., 2001a, 2008; Renosh et al., 2015)

The aim of this work is to characterize by MF analysis the image patterns in the wavelength range for the common bands of the satellites used, as well as both NDVI and EVI indexes. In order to investigate how the image information is affected by the sampling with different spatial and radiometric resolutions, we have also analysed images of the same site but acquired by two different satellites: Landsat- 7 and Ikonos-2.

We present a comparative analysis of multi-fractal (MF) tools applied to multi-spectral images obtained by Ikonos-2 and LANDSAT-7. Both satellites have several bands in visible and near-infrared spectral regions in common that can be used in vegetation-index estimation. However, the bands have different spatial resolution ( $4 \mathrm{~m}$ for Ikonos- 2 and $30 \mathrm{~m}$ for LANDSAT-7), and radiometric resolution (11 bits for Ikonos-2 and 8 bits for LANDSAT-7). The bands we have chosen are red, green, blue and near infrared. For each of those bands, the MF spectrum has been calculated directly from the Hölder exponents $\alpha$ and the singularity spectrum $\mathrm{f}(\alpha)$. The same calculations were applied for NDVI and EVI estimated on red, blue and near-infrared bands for each image.

\section{Materials and methods}

\subsection{Images}

As already noted, in this work we have analysed two images of the same site acquired from different satellites, Landsat7 and Ikonos-2. Both are multi-spectral images with several bands that cover several regions of the electromagnetic spectrum in the visible and near-infrared wavelength.

Landsat-7 was put in orbit in April 1999. This satellite follows a sun-synchronous orbit at $705 \mathrm{~km}$ of altitude, with an equatorial crossing time of 10:00 LT in the descending node. It requires $98.8 \mathrm{~min}$ to circle the Earth, tracing a worldwide reference system (WRS) of just over 230 ground paths. Over at least three decades, Landsat-7 orbits over each of these paths once every 16 days in a repetitive cycle (Mika, 1997).

The main Landsat-7 sensor for Earth observation is the Enhanced Thematic Mapper Plus (ETM+). The ETM+ operates as a whiskbroom scanner and acquires data for seven spectral bands: visible (ETM+\#1, from 0.45 to $0.52 \mu \mathrm{m}$; $\mathrm{ETM}+\# 2$, from 0.53 to $0.61 \mu \mathrm{m}$; ETM+\#3, from 0.63 to $0.69 \mu \mathrm{m})$, near infrared (ETM+\#4, from 0.78 to $0.9 \mu \mathrm{m}$ ), shortwave infrared (ETM+\#5, from 1.55 to $1.75 \mu \mathrm{m}$, and $\mathrm{ETM}+\# 7$, from 2.09 to $2.35 \mu \mathrm{m}$ ) and thermal infrared (ETM+\#6, from 10.4 to $12.5 \mu \mathrm{m})$. The ETM+ ground sampling distance (pixel size in the images) is $30 \mathrm{~m}$ for the six reflective bands and $60 \mathrm{~m}$ for the thermal band. The ETM+ also acquires images for a panchromatic band (ETM+\#8, from 0.52 to $0.9 \mu \mathrm{m}$ ) with a $15 \mathrm{~m}$ ground sampling distance. The radiometric resolution of the Landsat- 7 data is 8 bits per pixel or 256 grey levels for the pixel digital value.

Ikonos-2 was launched in September 1999. Its panchromatic sensor, with a resolution of $0.82 \mathrm{~m}$, provided the first very high-resolution images of the Earth's surface from earth observation satellites (EOS). The Ikonos-2 orbiting altitude is approximately $681 \mathrm{~km}$; it is inclined $98.1^{\circ}$ to the equator and it provides sun-synchronous operation. The equato- 
rial crossing time of Ikonos-2 is 10:30 LT in the descending node. The orbit provides daily access to sites within $45^{\circ}$ of nadir (Dial et al., 2003).

The multi-spectral sensor simultaneously collects blue (IK\#1, from 0.445 to $0.516 \mu \mathrm{m}$ ), green (IK\#2, from 0.506 to $0.595 \mu \mathrm{m}$ ), red (IK\#3, from 0.632 to $0.698 \mu \mathrm{m}$ ) and nearinfrared (IK\#4, from 0.757 to $0.853 \mu \mathrm{m}$ ) bands with $3.28 \mathrm{~m}$ resolution at nadir. Both images, panchromatic and multispectral, have a radiometric resolution of 11 bits per pixel or 2048 grey levels for the pixel digital value.

The Landsat-7 multi-spectral image used in this study was acquired on 6 August 2000 at 10:46 LT. and it corresponds to the scene with WRS coordinates: path and row 201 and 32 , respectively. This scene is located in the central region of Spain and it covers a square surface of approximately $180 \mathrm{~km}$ side length, located around Madrid. Solar azimuth and elevation angles for this scene are 132.44 and $58.62^{\circ}$ respectively.

The Ikonos-2 datum used in this study is a multi-spectral image acquired on 8 August 2000 at 11:03 LT. It covers an area of $11 \mathrm{~km}^{2}$ located near Aranjuez, south of Madrid, in the central region of Spain. Solar azimuth and elevation angles for this scene are 139.5 and $60.79^{\circ}$ respectively. Both images were corrected geometrically to the same cartographic projection: Universal Transverse Mercatorprojection (UTM), zone $30^{\circ} \mathrm{N}$, by a co-registration process.

The analysis has been carried out on a subset that covers (approximately) the same area in both the Landsat-7 and the Ikonos-2 images, corresponding to a region located north of the town of Aranjuez. The representative elements of the land used in the selected area are irrigation crops, pastures, heaths, unirrigated land cultivations and olive groves. The Landsat-7 subset image is a square of $512 \times 512$ pixels with a resolution of $30 \mathrm{~m}$ covering a somewhat larger surface than the Ikonos2 image. The Ikonos- 2 image consists of a square subset with $2048 \times 2048$ pixels and $4 \mathrm{~m}$ resolution.

\subsection{Vegetation indexes}

Vegetation is one of the landscape elements that has received the most attention in the field of image analysis. Therefore, there are many parameters that can be used to obtain information on vegetation from remote sensing imagery.

One of the main parameters is made up of the vegetation indexes. These indexes allow to detect the presence of vegetation in an area and its activity, since its values are related to this activity. For this, we can use the reflectance values corresponding to the different wavelengths, interpreting these in relation to the photosynthetic activity. Of these indexes, the most commonly used is the NDVI.

The NDVI is defined by

$\mathrm{NDVI}=\frac{\mathrm{NIR}-\mathrm{R}}{\mathrm{NIR}+\mathrm{R}}$,

where NIR is the pixel value in the near-infrared band and $\mathrm{R}$ the pixel value in the red band. The values of this in- dex are within the range $(-1,1)$ and their positive values are sensitive to the proportion of soil and vegetation in each pixel (Carlson and Ripley, 1997). Pixels with NDVI $<0.2$ are considered without vegetation or bare soil. Pixels with NDVI $>0.5$ are considered as fully covered by vegetation.

EVI, the other vegetation index, is defined by

$\mathrm{EVI}=2.5 \frac{(\mathrm{NIR}-\mathrm{R})}{\left(L+\mathrm{NIR}+C_{1} \mathrm{R}-C_{2} \mathrm{~B}\right)}$,

where NIR is the pixel value in the near-infrared band, $R$ the pixel value in the red band and $B$ the pixel value in the blue band. $L, C_{1}$ and $C_{2}$ are constants with the values 1, 6 and 7.5 respectively. The main characteristic of this index is that it corrects some distortions caused by the light dispersion from aerosols, as well as the background soil (Huete et al., 2014).

\subsection{Multi-fractal image analysis}

A monofractal object can be measured by counting the number $N$ of $\delta$ size boxes needed to cover the object. The measure depends on the box size as

$N(\delta) \propto \delta^{-D_{0}}$,

where

$D_{0}=\lim _{\delta \rightarrow 0} \frac{\log N(\delta)}{\log \frac{1}{\delta}}$

is the fractal dimension. $D_{0}$ is calculated from slope of a $\log -\log$ plot. However, many examples are found where a single scaling law cannot be applied and it is necessary to do a multi-scaling analysis.

There are several methods for implementing multi-fractal analysis. The universal multi-fractal (UM) model assumes that multi-fractals are generated from a random variable with an exponentiated extreme Levy distribution (Lavallée et al., 1991; Tessier et al., 1993). In UM analysis, the scaling exponent $K(q)$ is highly relevant. This function for the moments $q$ of a cascade conserved process is obtained according to Schertzer and Lovejoy (1987), as follows:

$K(q)=\left\{\begin{array}{l}\frac{C_{1}\left(q^{\alpha_{\mathrm{L}}}-q\right)}{\alpha_{\mathrm{L}}-1} \text { if } \alpha_{\mathrm{L}} \neq 1 \\ C_{1} q \log (q) \text { if } \alpha_{\mathrm{L}}=1\end{array}\right\}$,

where $C_{1}$ is the mean intermittency codimension and $\alpha_{\mathrm{L}}$ is the Levy index. These are known as the UM parameters.

Other method is the moment method developed by Halsey et al. (1986) and applied to this case study. This method mainly uses three functions: $\tau(q)$, known as the mass exponent function; $\alpha$, the coarse Hölder exponent; and $f(\alpha)$, multi-fractal spectrum (MFS). A measure (or field), defined in two-dimensional image embedding space $(n \times n$ pixels $)$ and with values based on grey tones (for 8 bits, from 0 to 255), cannot be considered as a geometrical set and therefore cannot be characterized by a single fractal dimension. 
To characterize the scaling property of a variable measured on the spatial domain of the studied area, it divides the image into a number of self-similar boxes. Applying disjoint covering by boxes in an "up-scaling" partitioning process we obtain the partition function $\chi(q, \delta)$ (Feder, 1989) defined as follows:

$\chi(q, \delta)=\sum_{i=1}^{N(\delta)} \mu_{i}^{q}(\delta)=\sum_{i=1}^{N(\delta)} m_{i}^{q}$,

where $m$ is the mass of the measure, $q$ is the mass exponent, $\delta$ is the length size of the box and $N(\delta)$ is the number of boxes in which $m_{i}>0$. Based on this, the mass exponent function $\tau(q)$ shows how the moments of the measure scales with the box size:

$\tau(q)=\lim _{\delta \rightarrow 0} \frac{\log <\chi(q, \delta)>}{\log (\delta)}=\lim _{\delta \rightarrow 0} \frac{\log <\sum_{i=1}^{N(\delta)} m_{i}^{q}>}{\log (\delta)}$,

where $<>$ represents a statistical moment of the measure $\mu_{i}(\delta)$ defined on a group of non-overlapping boxes of the same size partitioning as the area studied.

The singularity index, $\alpha$, can be determined by the Legendre transformation of the $\tau(q)$ curve (Halsey et al., 1986) as follows:

$\alpha(q)=\frac{\mathrm{d} \tau(q)}{\mathrm{d} q}$.

The number of cells of size $\delta$ with the same $\alpha, N_{\alpha}(\delta)$, is related to the cell size as $N_{\alpha}(\delta) \propto \delta^{-f(\alpha)}$, where $f(\alpha)$ is a scaling exponent of the cells with common $\alpha$. Parameter $f(\alpha)$ can be calculated as follows:

$f(\alpha)=q \alpha(q)-\tau(q)$.

MFS, shown as plot of $\alpha$ versus $f(\alpha)$, quantitatively characterizes variability of the measure studied with asymmetry to the right and left indicating domination of small and large values respectively (Evertsz and Mandelbrot, 1992). There are three characteristic values obtained from MFS, the singularity $\alpha(q)$ values for $q=\{0,1,2\}$. The first value $(\alpha(0))$ corresponds to the maximum of MFS and it is related to the boxcounting dimension of the measure support; the second value is related to information or entropy dimension $(\alpha(1))$ and the third with the correlation dimension. The entropy dimension quantifies the degree of disorder present in a distribution. According to Andraud et al. (1994) and Gouyet (1996), a $\alpha$ (1) value close to 2.0 characterizes a system uniformly distributed throughout all scales, whereas a $\alpha(1)$ close to 0 reflects a subset of the scale in which the irregularities are concentrated. These three values will be shown from each calculation of MFS.

The width of the MF spectrum $(\Delta)$ indicates overall variability (Tarquis et al., 2001, 2014) and we have split it in two sections. Section I correspond to values $\alpha(q)<\alpha(0)$ or $q>0$ and section II to values with $\alpha(q)>\alpha(0)$ or $q<0$. In section I the amplitude, or semi-width, was calculated with differences $\Delta^{+}=\alpha(0)-\alpha(+5)$, and in section II with $\Delta^{-}=\alpha(-5)-\alpha(0)$.

To study the asymmetry of the MFS we have chosen the asymmetry index (AI) estimated as follows (Xie et al., 2010):

$\mathrm{AI}=\frac{\Delta \alpha_{\mathrm{L}}-\Delta \alpha_{\mathrm{R}}}{\Delta \alpha_{\mathrm{L}}+\Delta \alpha_{\mathrm{R}}} \quad \begin{aligned} & \Delta \alpha_{\mathrm{L}}=\alpha_{0}-\alpha_{\min } \\ & \Delta \alpha_{\mathrm{R}}=\alpha_{\max }-\alpha_{0}\end{aligned}$.

In our case, $\alpha_{0}$ is the singularity for $q=0$ or $\alpha(0), \alpha_{\min }$ is $\alpha(+5)$ and $\alpha_{\max }$ is $\alpha(-5)$. Therefore, we can rewrite the AI as follows:

$\mathrm{AI}=\frac{\Delta^{+}-\Delta^{-}}{\Delta^{+}+\Delta^{-}}$.

Expressing AI as Eq. (11), we can see that it is a normalized index based on the amplitudes $\Delta^{+}$and $\Delta^{-}$.

There are several works relating the UM model and the multi-fractal formalism based on $\tau(q)$ (Gagnon et al., 2003; Aguado et al., 2014; Morató et al., 2017, among others) through the following equations:

$f(\alpha)=E-c(\gamma) ; \alpha=E-\gamma$,

$\tau(q)=E(q-1)-K(q)$,

where $E$ is the Euclidean dimension in which the measure is embedded, in this case $E=2$, and $c(\gamma)$ is the codimension of the singularity of the density of the multi-fractal measure $\gamma$.

\section{Results and discussion}

\subsection{Radiometric influence in the multi-fractal spectrum}

To study the influence of radiometric resolution on Ikonos2 image information complexity, the original pixel code (11 bits) has been transformed to 8 bits through a rescaling based on minimum and maximum values between 0 and 255 , with the aim of preserving the initial histogram shape.

We first discuss the results obtained for the $2048 \times 2048$ pixel Ikonos-2 image shown in Fig. 1, in bands combination of false colour (IK\#4, IK\#3, IK\#2 band combination in RGB visualization). In Fig. 2 IK\#1, IK\#2, IK\#3 and IK\#4 band histograms are shown. In the right column are histograms with the original radiometric resolution and in the left column the corresponding histograms are rescaled to 8 bits. The histograms present a bimodal structure with a narrow peak of low-value pixels (dark grey) showing a sharp maximum and a wider peak around a second lower maximum. For bands IK\#1, IK\#2 and IK\#3, the narrow peak maximum corresponds to vegetation, mainly irrigation crops, showing strong water absorption. This effect is particularly important in band IK\#3. High-value pixels (lighter grey) correspond to ground zones with lower vegetation content. However, as 


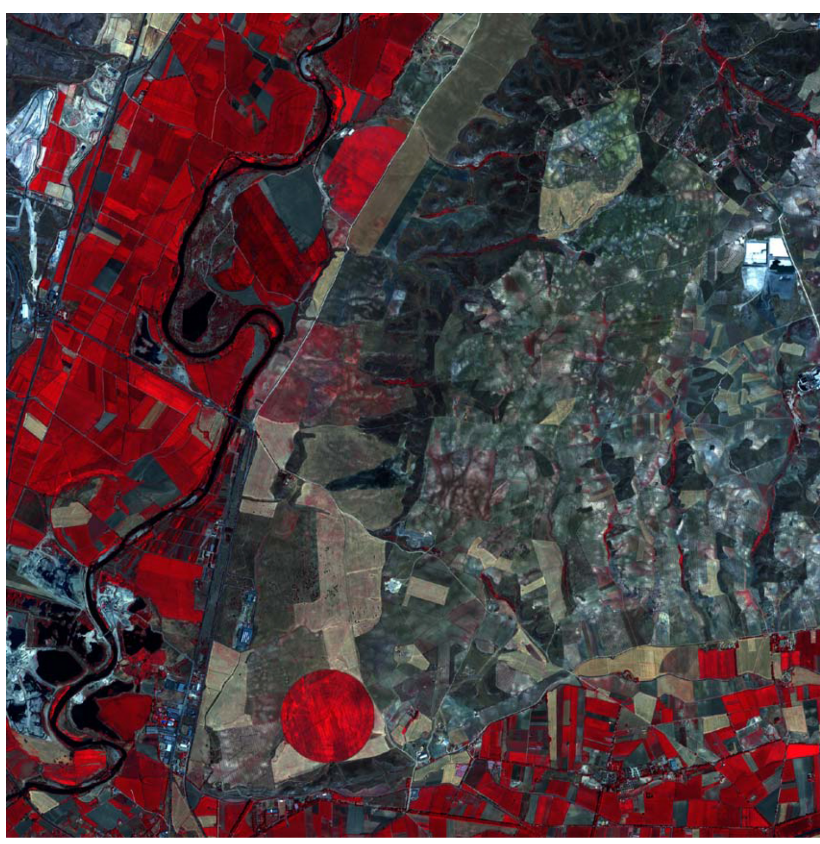

Figure 1. The Ikonos-2 image in band combinations of false colour (IK\#4, IK\#3 and IK\#2 in RGB). The image has a size of $2048 \times 2048$ pixels, each area unit corresponding to $4 \times 4 \mathrm{~m}$. The coordinates UTM (zone 30) of the upper left and lower right pixel in the image are: $\mathrm{ULX}=446037 \mathrm{~m}, \mathrm{ULY}=4441684 \mathrm{~m}, \mathrm{LRX}=454229 \mathrm{~m}$ and $\mathrm{LRY}=4433492 \mathrm{~m}$.

vegetation shows high reflectivity in the near-infrared, IK\#4 band histogram shows a predominance of high-value pixels (lighter grey pixels) corresponding to dense vegetation parts. For both radiometric resolutions the shapes of the histograms are very similar, as it was our intention (see Fig. 2).

We cover the image with boxes of size $\delta=2^{-n}$ and we change the box size from 2048 to 2 pixels, that is, $\delta=$ $2048 / 2^{n}$ with $n=0,1,2, \ldots, 10$. For each value of the parameter $q$, from -5 to +5 with increments of 0.5 , the partition function (Eq. 6) is computed and $\log \chi(q, \delta)$ versus $\log \delta$ is plotted in Fig. 3. Each graph contains 11 points and from these a range of scales are selected for the least-square linear fit reaching the maximum possible scales and with a standard error in the slope, the estimated values of $\tau(q)$, less than 0.01. Then, using Eqs. (7)-(8), $\alpha(q)$ and $f(\alpha)$ are obtained. Comparing the range of scales used in both radiometric resolutions, the bands using the original data (11 bits) showed a wider range of scales for the linear fit, up to 4 pixels, whereas in the 8-bit radiometric resolution were required up to 32 pixels (see arrows in Fig. 3).

The MF spectra $f(\alpha)$ corresponding to the four bands of multi-spectral Ikonos images are shown in Fig. 4. These differences found in the multi-scaling behaviour of each band are in agreement with previous works (Cheng, 2004; Lovejoy et al., 2008). Just by visual observation, there is a remarkable difference between the bands \#3 and \#4, and red and NIR, between 8 and 11 bits respectively. Higher radiometric resolution gives a higher range of possible grey values per pixel. Note that this radiometric resolution effect is manifested in both sections of the MF spectra (for $q>0$ and for $q<0$ ).

Some characteristic parameters obtained from these MF spectra are shown in Table 1 and Table 2. As expected, in both radiometric resolutions and in each band the $\alpha(0)$ is practically 2 , as the measure is defined in the entire plane and it has an Euclidean dimension of 2. With respect to the $\alpha$ (1) value, certain differences are found. Comparing the bands in 8 bits to the same ones in 11 bits, the entropy dimension was always higher. However, considering the standard errors, only IK\#1 (B) and IK\#2 (G) bands were significantly different, with the blue band showing the highest difference. Meanwhile, red and NIR bands are not significantly different. This shows that a more spatially uniform distribution for the bands of Ikonos- 28 bits than in 11 bits. The same behaviour is observed in the $\alpha$ (2).

The amplitudes calculated $\left(\Delta^{+}\right.$and $\left.\Delta^{-}\right)$in Ikonos-2 11bit bands present opposite trends (Table 1). Note that amplitude $\Delta^{+}$decreases as band wavelength grows, whereas the other amplitude $\Delta^{-}$diminishes. Observing these parameters in Ikonos-2 8-bit bands (Table 2) a different trend and behaviour are found. In this case both $\Delta^{+}$and $\Delta^{-}$increase as the wavelength increases for the three visible bands, but decrease for the near-infrared band (IK\#4).

The AI estimated on these MFS amplitudes on each radiometric resolution are shown in the last column of Table 1 and Table 2. Comparing the bands in 8 bits to the same ones in 11 bits, the behaviour is similar: there is a decreasing trend from IK\#1 to IK\#4, although the range of values is different. At a resolution of 11 bits from a positive $\mathrm{AI}=0.240$ at blue band goes to a negative $\mathrm{AI}=-0.237$ at NIR band. On the other hand, at a resolution of 8 bits, an $\mathrm{AI}=0.092$ goes to a negative $\mathrm{AI}=-0.347$. The more symmetric MFS are found in green and red bands at a resolution of 11 bits and in blue and green bands at a resolution of 8 bits.

Doing the same study for the vegetation indexes we found the following. The bi-log plot of the partition function $(\chi(q, \delta))$ versus $\delta$ is plotted in Fig. 5 for both vegetation indexes at both radiometric resolutions. Each graph contains 11 points as the bands from where they were estimated. The linear fit was done with the same methodology as that for the four bands. In this case only the EVI at 8 bits shows a better linear trend in a wider range of scales. However, to better compare both vegetation indexes, from both radiometric resolutions, a range achieving 32 pixels $(128 \mathrm{~m})$ was selected as shown by arrows in Fig. 5 .

The MF spectra $f(\alpha)$ of EVI and NDVI estimated for both radiometric resolutions of Ikonos images are shown in Fig. 4. Both vegetation indexes show differences due to the transformation from 11 to 8 bits. However, NDVI shows higher differences in the MFS, mainly in the part corresponding to $q$ negative values (right side). Even EVI presents changes; its MFS is closer at both radiometric resolutions. Compar- 

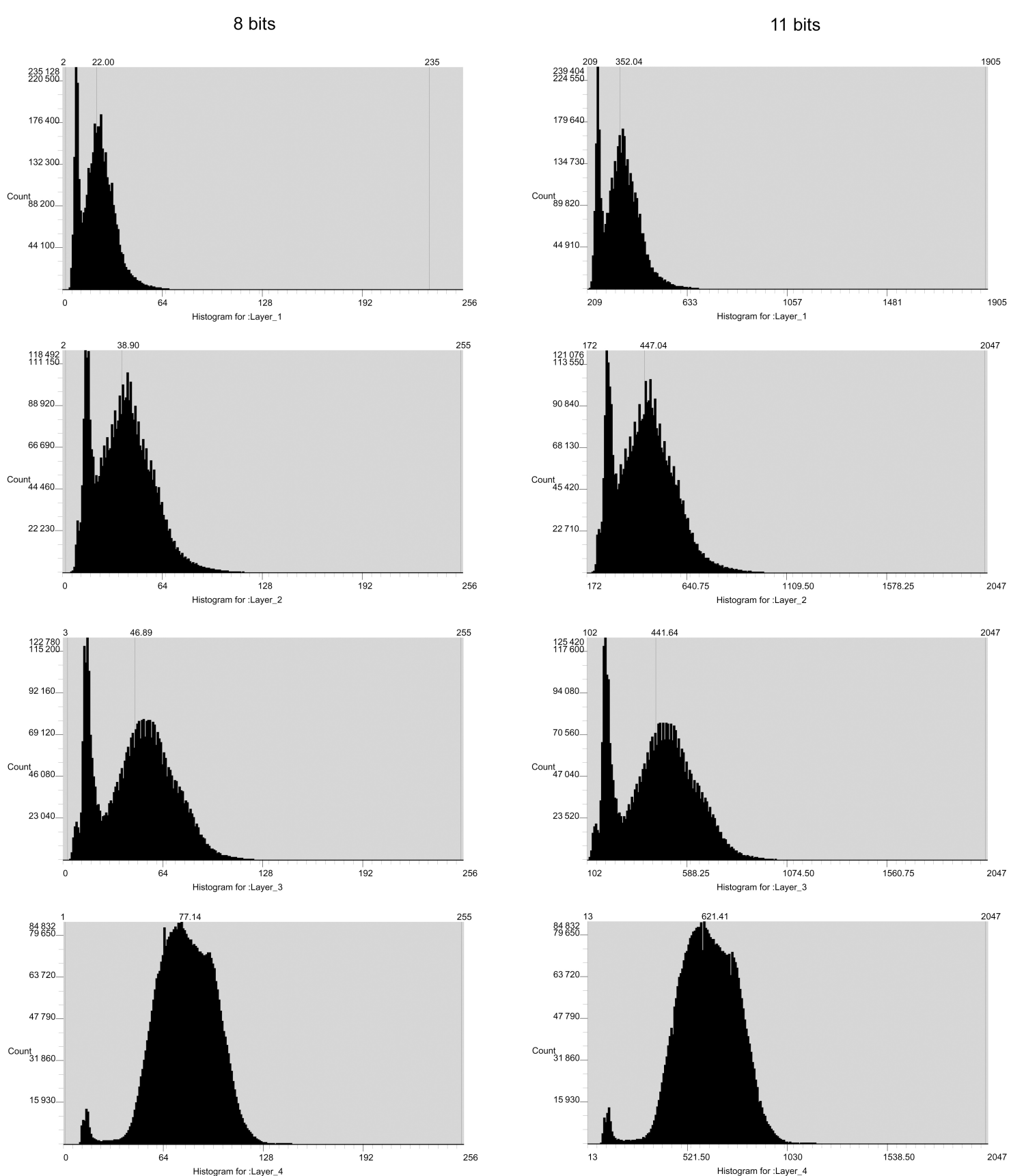

Figure 2. Histograms of the four bands of the Ikonos-2 image for the original radiometric resolution, 11 bits (right), and the minimummaximum rescaled 8-bit radiometric resolution (left).

ing the range of $f(\alpha)$ values in the vegetation indexes to the range obtained in the four bands (left column in Fig. 4), there is a remarkable contrast. Meanwhile the NIR band of 8 bits achieves a $f(\alpha)$ value close to 0.5 ; EVI and NDVI achieve values closer to 0.2 . These differences are higher in the 11-bit image; the red band achieves a $f(\alpha)$ value close to 0.9 and vegetation indexes again achieve values of $\sim 0.2$. The same characteristic parameters obtained from the band MF spectra were calculated for the vegetation indexes and are shown in Table 1 and Table 2.

With respect to the $\alpha(1)$ values, certain differences are found between the vegetation indexes. Comparing the NDVI in 8 bits to the same ones in 11 bits, the entropy dimension was always higher than it was found to be in the bands. However, EVI shows the contrary: entropy values of the 11-bit image are higher than the 8-bit image, although the differences are not significant. Therefore, the radiometric resolution af- 
Table 1. Parameters obtained from the multi-fractal spectrum from each band of the Ikonos-2 image, and the vegetation indexes (VIs) estimated, with a pixel size of $4 \mathrm{~m}$ and a radiometric resolution of 11 bits. The amplitudes of $\alpha$ values are presented as $\Delta^{+}$and $\Delta^{-}$ corresponding to $\alpha(0)-\alpha(5)$ and $\alpha(-5)-\alpha(0)$ respectively. The asymmetry index (AI) corresponds to $\frac{\Delta^{+}-\Delta^{-}}{\Delta^{+}+\Delta^{-}}$.

\begin{tabular}{|c|c|c|c|c|c|c|}
\hline & Band & $q$ & $\alpha(q)$ & $\Delta^{+}$ & $\Delta^{-}$ & $\mathrm{AI}$ \\
\hline \multirow{13}{*}{ Ikonos-2 (11 bits) } & & 0 & $2.001 \pm 0.001$ & \multirow{3}{*}{0.418} & \multirow{3}{*}{0.256} & \multirow{3}{*}{0.240} \\
\hline & IK\#1 & 1 & $1.938 \pm 0.005$ & & & \\
\hline & & 2 & $1.865 \pm 0.009$ & & & \\
\hline & \multirow{3}{*}{$\mathrm{IK} \# 2$} & 0 & $2.001 \pm 0.001$ & \multirow{3}{*}{0.377} & \multirow{3}{*}{0.313} & \multirow{3}{*}{0.093} \\
\hline & & 1 & $1.936 \pm 0.005$ & & & \\
\hline & & 2 & $1.871 \pm 0.007$ & & & \\
\hline & \multirow{3}{*}{ IK\#3 } & 0 & $2.001 \pm 0.001$ & \multirow{3}{*}{0.348} & \multirow{3}{*}{0.382} & \multirow{3}{*}{-0.047} \\
\hline & & 1 & $1.937 \pm 0.005$ & & & \\
\hline & & 2 & $1.878 \pm 0.006$ & & & \\
\hline & \multirow{3}{*}{ IK\#4 } & 0 & $2.001 \pm 0.001$ & \multirow{3}{*}{0.290} & \multirow{3}{*}{0.470} & \multirow{3}{*}{-0.237} \\
\hline & & 1 & $1.959 \pm 0.005$ & & & \\
\hline & & 2 & $1.908 \pm 0.009$ & & & \\
\hline & VI & $q$ & $\alpha(q)$ & $\Delta^{+}$ & $\Delta^{-}$ & $\mathrm{AI}$ \\
\hline \multirow{6}{*}{ Ikonos-2 (11 bits) } & & 0 & $2.000 \pm 0.001$ & \multirow{3}{*}{0.516} & \multirow{3}{*}{1.166} & \multirow{3}{*}{-0.386} \\
\hline & NDVI & 1 & $1.886 \pm 0.008$ & & & \\
\hline & & 2 & $1.779 \pm 0.010$ & & & \\
\hline & \multirow{3}{*}{ EVI } & 0 & $2.000 \pm 0.001$ & \multirow{3}{*}{0.270} & \multirow{3}{*}{0.877} & \multirow{3}{*}{-0.533} \\
\hline & & 1 & $1.948 \pm 0.002$ & & & \\
\hline & & 2 & $1.897 \pm 0.004$ & & & \\
\hline
\end{tabular}

Table 2. Parameters obtained from the multi-fractal spectrum from each band of the Ikonos-2 image, and the vegetation indexes (VIs) estimated, with a pixel size of $4 \mathrm{~m}$ and a radiometric resolution of 8 bits. The amplitudes of $\alpha$ values are presented as $\Delta^{+}$and $\Delta^{-}$corresponding to $\alpha(0)-\alpha(5)$ and $\alpha(-5)-\alpha(0)$ respectively. The asymmetry index (AI) corresponds to $\frac{\Delta^{+}-\Delta^{-}}{\Delta^{+}+\Delta^{-}}$.

\begin{tabular}{|c|c|c|c|c|c|c|}
\hline & Band & $q$ & $\alpha(q)$ & $\Delta^{+}$ & $\Delta^{-}$ & AI \\
\hline \multirow{13}{*}{ Ikonos-2 (8 bits) } & & 0 & $2.000 \pm 0.001$ & \multirow{3}{*}{0.231} & \multirow{3}{*}{0.192} & \multirow{3}{*}{0.092} \\
\hline & IK\#1 & 1 & $1.971 \pm 0.003$ & & & \\
\hline & & 2 & $1.930 \pm 0.006$ & & & \\
\hline & \multirow{3}{*}{ IK\#2 } & 0 & $2.000 \pm 0.001$ & \multirow{3}{*}{0.270} & \multirow{3}{*}{0.287} & \multirow{3}{*}{-0.031} \\
\hline & & 1 & $1.963 \pm 0.004$ & & & \\
\hline & & 2 & $1.914 \pm 0.006$ & & & \\
\hline & \multirow{3}{*}{ IK\#3 } & 0 & $2.000 \pm 0.001$ & \multirow{3}{*}{0.323} & \multirow{3}{*}{0.614} & \multirow{3}{*}{-0.311} \\
\hline & & 1 & $1.945 \pm 0.005$ & & & \\
\hline & & 2 & $1.887 \pm 0.006$ & & & \\
\hline & \multirow{3}{*}{ IK\#4 } & 0 & $2.000 \pm 0.001$ & \multirow{3}{*}{0.248} & \multirow{3}{*}{0.512} & \multirow{3}{*}{-0.347} \\
\hline & & 1 & $1.966 \pm 0.004$ & & & \\
\hline & & 2 & $1.923 \pm 0.008$ & & & \\
\hline & VI & $q$ & $\alpha(q)$ & $\Delta^{+}$ & $\Delta^{-}$ & AI \\
\hline \multirow{6}{*}{ Ikonos-2 (8 bits) } & & 0 & $2.000 \pm 0.002$ & \multirow{3}{*}{0.337} & \multirow{3}{*}{0.984} & \multirow{3}{*}{-0.490} \\
\hline & NDVI & 1 & $1.932 \pm 0.005$ & & & \\
\hline & & 2 & $1.855 \pm 0.008$ & & & \\
\hline & \multirow{3}{*}{ EVI } & 0 & $2.000 \pm 0.002$ & \multirow{3}{*}{0.300} & \multirow{3}{*}{0.874} & \multirow{3}{*}{-0.488} \\
\hline & & 1 & $1.940 \pm 0.004$ & & & \\
\hline & & 2 & $1.873 \pm 0.006$ & & & \\
\hline
\end{tabular}



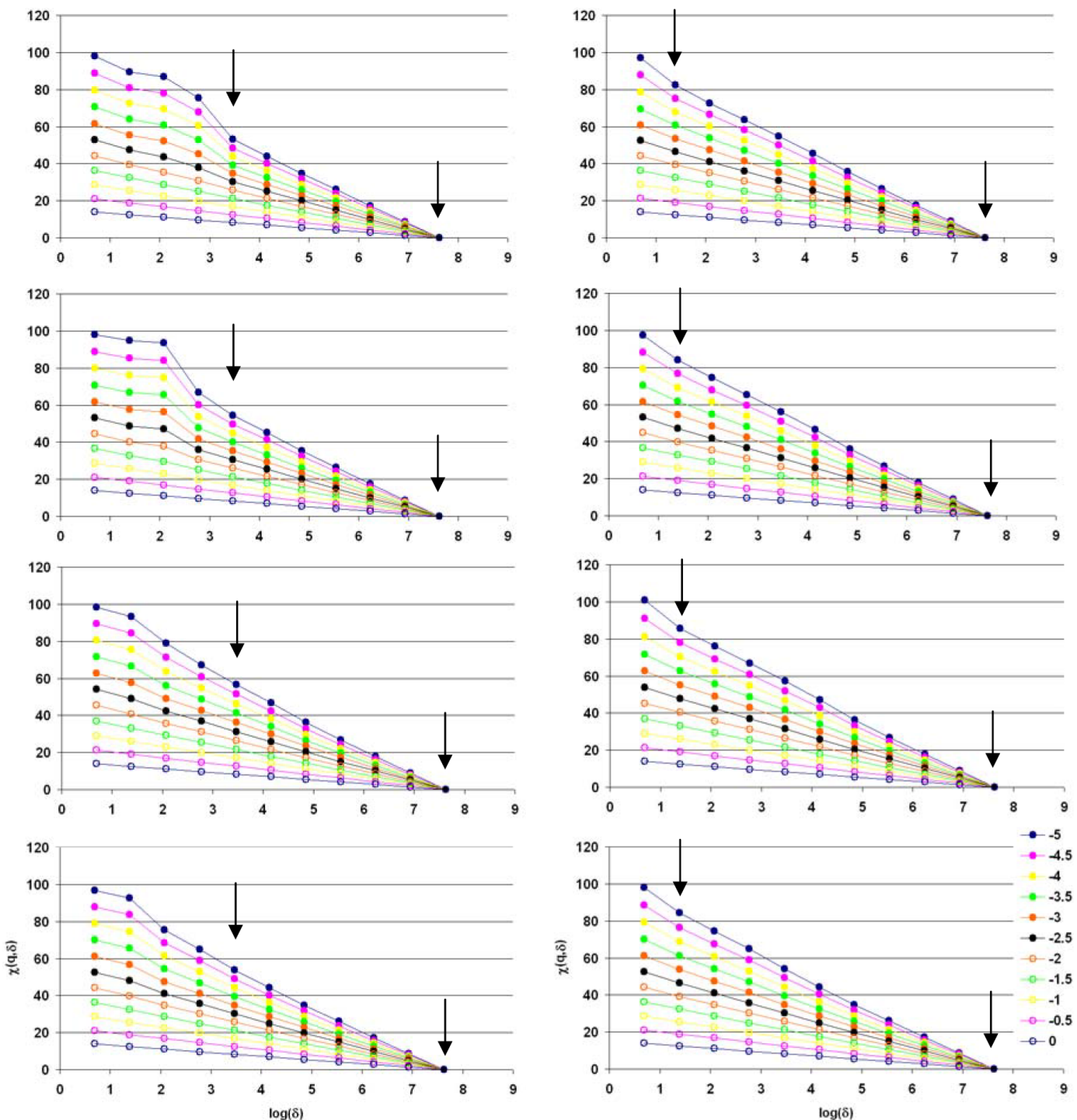

Figure 3. Bi-log plots of the partition function $\chi(q, \delta)$ versus $\delta$ for the first four bands of the Ikonos- 2 satellite and for $q<0$ values. From top to bottom we show the results for IK\#1, IK\#2, IK\#3 and IK\#4. The left column correspond to the 8-bit image and the right column to the 11-bit image. The arrows marked the range of scales used for the fit and to calculate the slope for different values of $q$ (7 points in the left column and 10 points in the right column).

fects NDVI more than EVI. The former presents a more uniform space distribution in 8 bits than in the 11-bit image. The same behaviour is observed in $\alpha(2)$.

The amplitudes calculated $\left(\Delta^{+}\right.$and $\left.\Delta^{-}\right)$in Ikonos-2 11bit vegetation indexes present a similar situation (Table 1). The amplitude $\Delta^{+}$is lower than amplitude $\Delta^{-}$and therefore the AI estimated is negative. This is visually perceived in Fig. 4 (right column). Observing these parameters in Ikonos28 bits vegetation indexes (Table 2), similar situations are found but the values are lower. In both images (11 and 8 bits) NDVI shows higher values for both amplitudes, $\Delta^{+}$and $\Delta^{-}$.

All the AI estimated for both vegetation indexes on each radiometric resolution are negative (Table 1 and Table 2), in- dicating a high asymmetry on the right part of the MFS, as shown in Fig. 4. Comparing the AI values in 8 bits to the same ones in 11 bits, they are similar which shows that the shape of the MFS is similar as this index is a normalized index. However, the values of the amplitudes mark a higher change in NDVI than in EVI.

\subsection{Spatial resolution influence in the multi-fractal spectrum}

A comparison is made between Landsat, with an original pixel code of 8 bits, and the rescaled histograms from Ikonos, with an original pixel code of 11 bits. In this section, we discuss the results obtained in the MF analysis on the $512 \times 512$ 

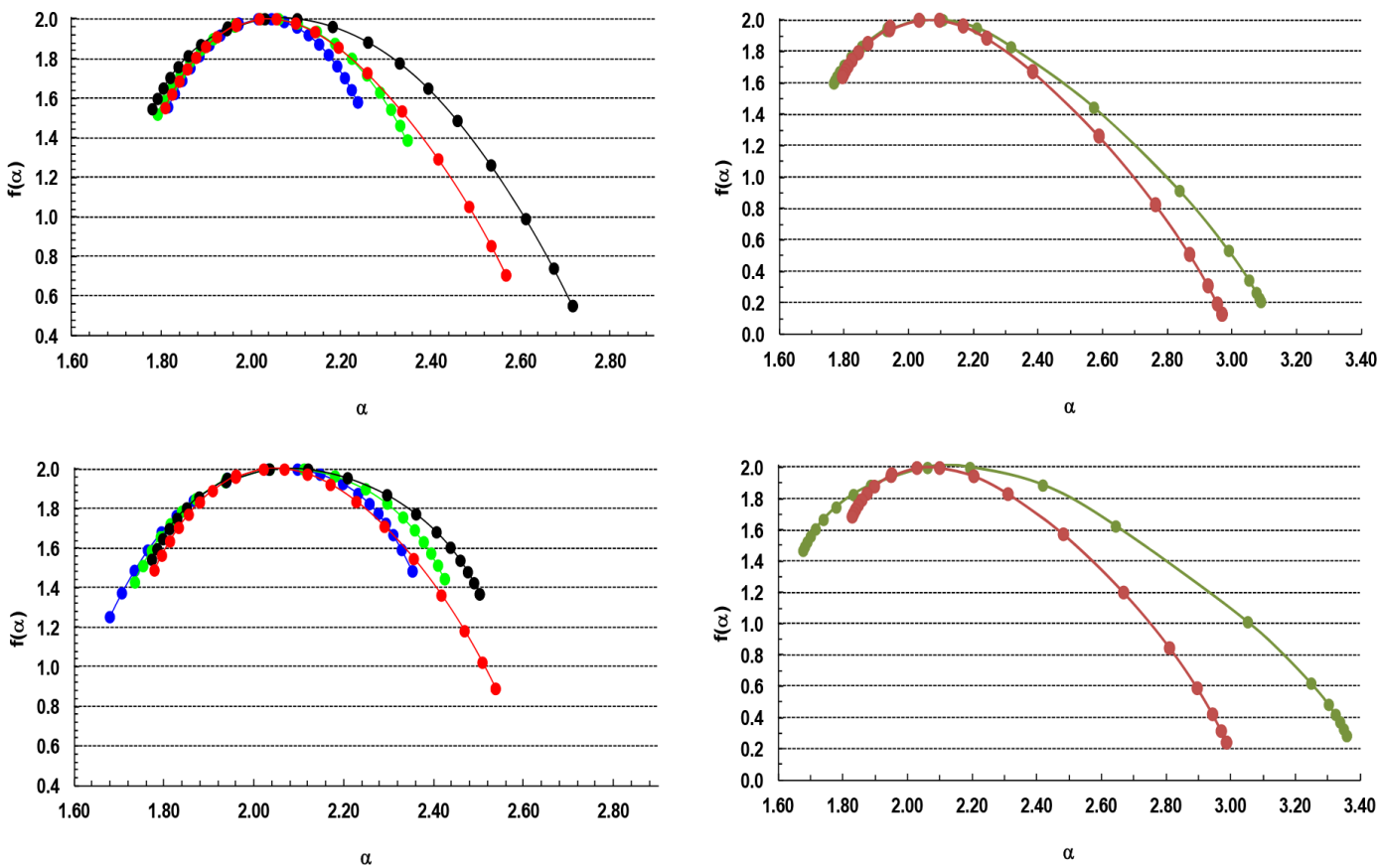

Figure 4. Multi-fractal spectrum of Ikonos-2 images for the original pixel values coded in 11 bits (lower) and the minimum-maximum rescaled to 8 bits (upper). Left column corresponds to each band analysed: IK\#1 in blue colour, IK\#2 in green colour, IK\#3 in red colour and IK\#4 in black. Right column corresponds to vegetation indexes: NDVI in green colour and EVI in brown.
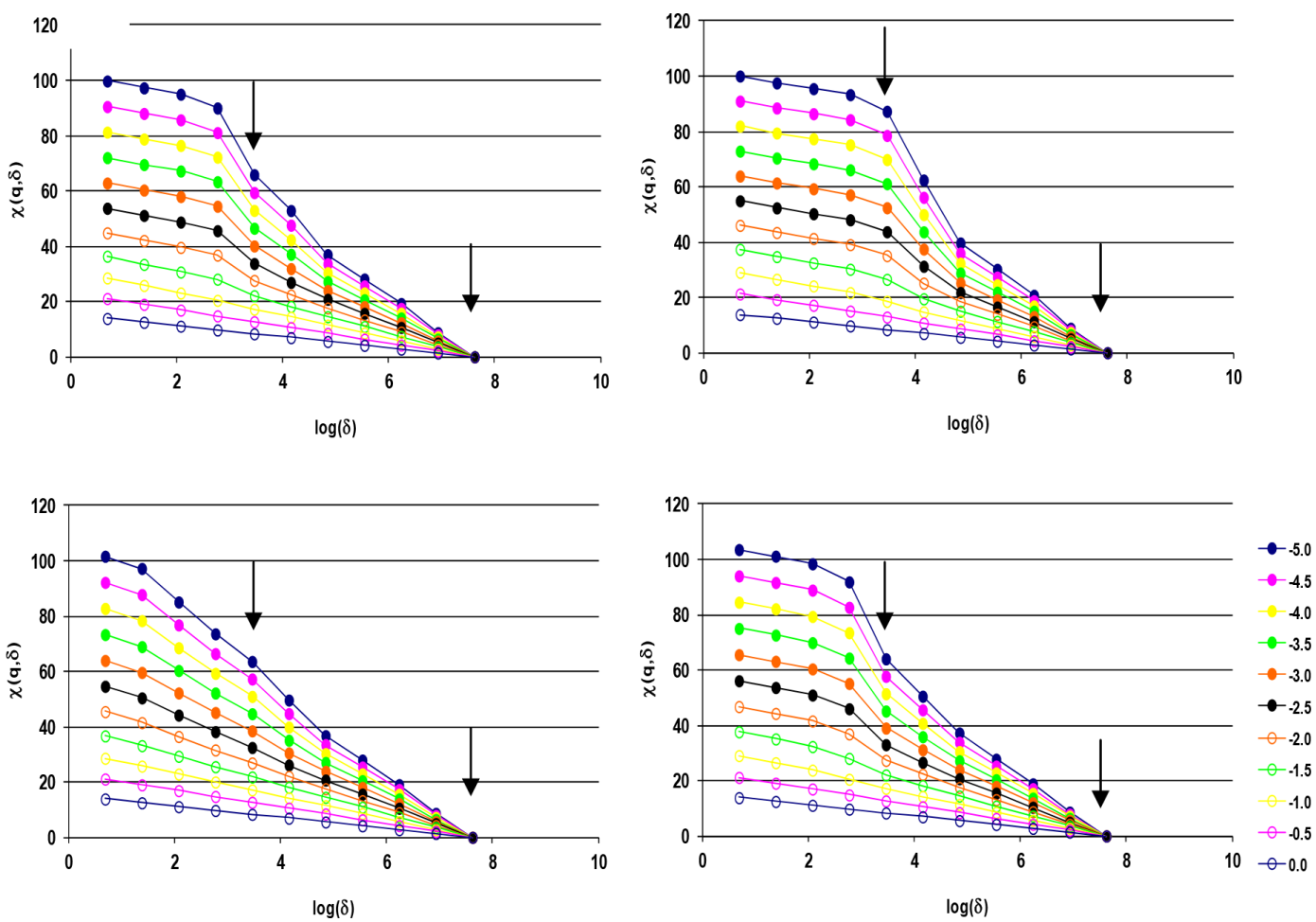

Figure 5. Bi-log plots of the partition function $\chi(q, \delta)$ versus $\delta$ for the vegetation indexes estimated from blue, red and NIR bands of the Ikonos- 2 satellite and for $q<0$ values. From top to bottom we show the results for NDVI and EVI respectively. The left column corresponds to the 8-bit image and the right column to the 11-bit image. The arrows marked the range of scales used for the fit and to calculate the slope for different values of $q$ (7 points). 


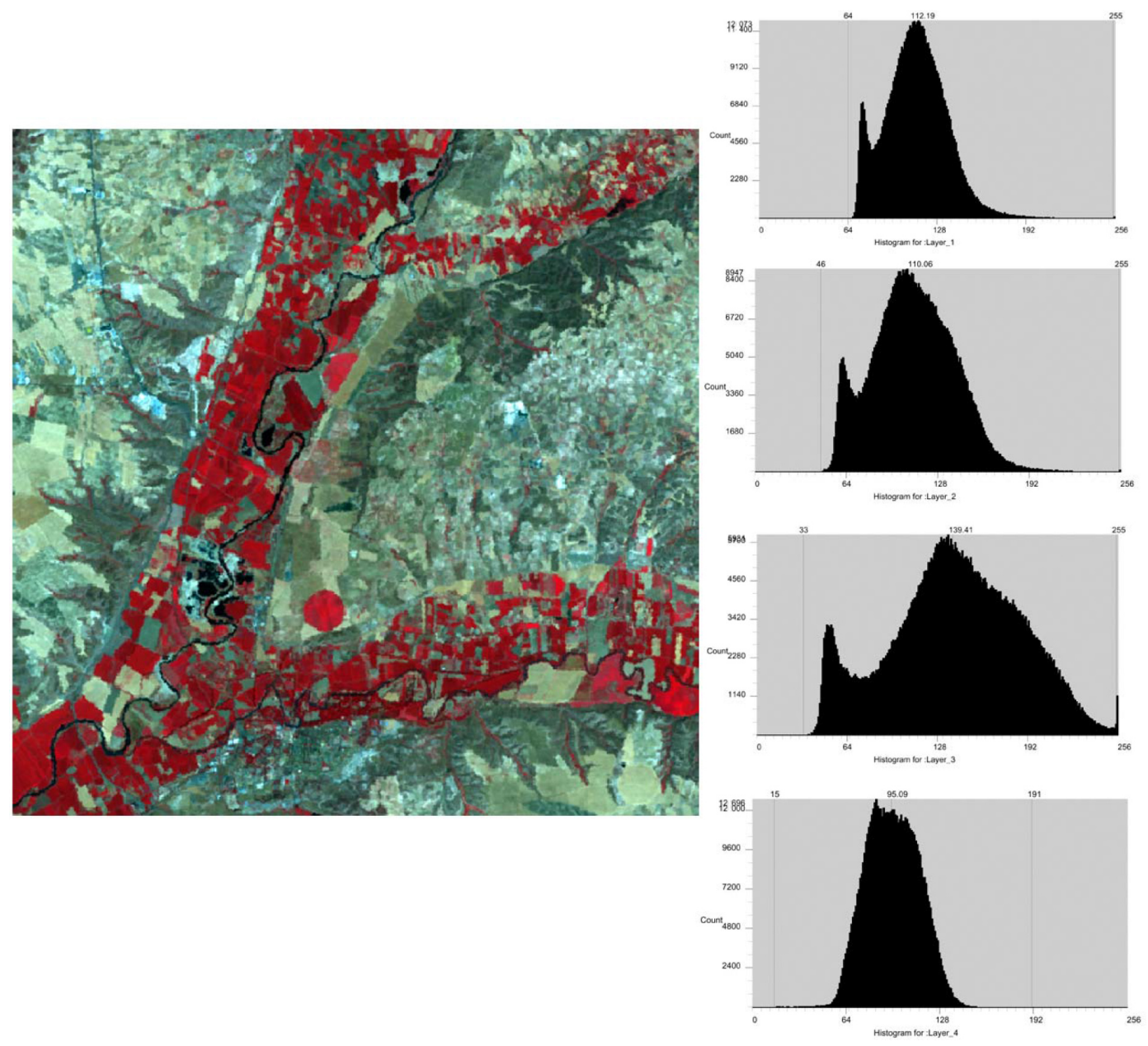

Figure 6. The Landsat-7 image and the histograms for the first four bands: blue (ETM+\#1), green (ETM+\#2), red (ETM+\#3) and near infrared (ETM+\#4). The image has a size of $512 \times 512$ pixels, each area unit corresponds to $30 \times 30 \mathrm{~m}$. The coordinates UTM (zone 30 ) of the upper-left and lower-right pixel in the image are ULX $=442185 \mathrm{~m}, \mathrm{ULY}=4445568 \mathrm{~m}, \mathrm{LRX}=457545 \mathrm{~m}$ and LRY $=4430208 \mathrm{~m}$.

pixel Landsat-7 image shown in Fig. 6, in bands combination of false colour (ETM+\#3, ETM+\#2 and ETM+\#1 band combination in RGB visualization).

In the right column of Fig. 6, the histograms of the Landsat-7 image for the first four bands are shown. The histograms present a bimodal structure, except for ETM+\#4 (NIR), in which there is only one peak. Comparing these histograms with those obtained for Ikonos-2 8-bit image (Fig. 2), the peaks are not so abrupt and narrow. At the same time, ETM+\#1, ETM+\#2 and ETM+\#3 bands show the absolute maximum peak at high-value pixels (light grey) and a second one at lower-value pixels (dark grey). These bands are more centred and do not show a shift to the left as the Ikonos28 -bit bands (IK\#1, IK\#2 and IK\#3. In the case of the NIR band, Landsat- 7 and Ikonos- 28 bits are quite similar except for the absence of a second peak.

In the calculations, box sizes range from 512 to 2 pixels, that is, $\delta=512 / 2^{n}$ with $n=0,1, \ldots, 8$. For each value of the parameters $q$, from -5 to +5 with increments of 0.5 , we compute the partition function, and the bi-log $\chi(q, \delta)$ versus $\delta$ is plotted in Fig. 7. In this case each linear fits contains only 9 points as the size of the image is $512 \times 512$ pixels. The same method was applied to select the range of scales used in the linear fit, achieving a scale of 4 pixels. Changing from pixels to metres, the scale achieved, used in Landsat- 7 in the MF analysis, was $\sim 120 \mathrm{~m}$. In the case of the Ikonos- 28 bits the scale was 32 pixels or $128 \mathrm{~m}$, very close to Landsat-7.

The MF spectra, $f(\alpha)$, corresponding to the first four bands of multi-spectral Landsat-7 images are shown in Fig. 8 . From a comparison of Figs. 4 and 8 we see that Landsat-7 image MF spectra are always located inside the corresponding Ikonos-2 MF spectra. For a given value of Hölder exponent $\alpha$, the relation $f_{\text {Landsat }}(\alpha) \leq f_{\text {Ikonos }}(\alpha)$ is always satisfied. This result means that Landsat-7 images show lower complexity than Ikonos-2 8-bit images. As stated in Sect. 2.1 Ikonos-2 satellite data are coded in 11 bits in contrast with Landsat-7 8-bit-coded data. To compare both sensors, with different spatial resolution, we pass Ikonos-2 from 11 to 
Table 3. Parameters obtained from the multi-fractal spectrum from each band of the Landsat-7 image, and the vegetation indexes (VIs) estimated, with a pixel size of $4 \mathrm{~m}$ and a radiometric resolution of 8 bits. The amplitudes of $\alpha$ values are presented as $\Delta^{+}$and $\Delta^{-}$corresponding to $\alpha(0)-\alpha(5)$ and $\alpha(-5)-\alpha(0)$ respectively. The asymmetry index (AI) corresponds to $\frac{\Delta^{+}-\Delta^{-}}{\Delta^{+}+\Delta^{-}}$.

\begin{tabular}{|c|c|c|c|c|c|c|}
\hline & Band & $q$ & $\alpha(q)$ & $\Delta^{+}$ & $\Delta^{-}$ & AI \\
\hline \multirow{5}{*}{ Landsat-7 } & ETM+\#1 & $\begin{array}{l}0 \\
1 \\
2\end{array}$ & $\begin{array}{l}2.001 \pm 0.001 \\
1.985 \pm 0.005 \\
1.960 \pm 0.010\end{array}$ & 0.160 & 0.119 & 0.147 \\
\hline & ETM+\#2 & $\begin{array}{l}0 \\
1 \\
2\end{array}$ & $\begin{array}{l}2.003 \pm 0.001 \\
1.988 \pm 0.004 \\
1.970 \pm 0.008\end{array}$ & 0.119 & 0.119 & 0.000 \\
\hline & ETM+\#3 & $\begin{array}{l}0 \\
1 \\
2\end{array}$ & $\begin{array}{l}2.001 \pm 0.001 \\
1.989 \pm 0.004 \\
1.974 \pm 0.007\end{array}$ & 0.095 & 0.110 & -0.073 \\
\hline & ETM+\#4 & $\begin{array}{l}0 \\
1 \\
2\end{array}$ & $\begin{array}{l}2.017 \pm 0.001 \\
1.989 \pm 0.004 \\
1.973 \pm 0.008\end{array}$ & 0.106 & 0.104 & 0.010 \\
\hline & VI & $q$ & $\alpha(q)$ & $\Delta^{+}$ & $\Delta^{-}$ & AI \\
\hline \multirow{2}{*}{ Landsat-7 } & NDVI & $\begin{array}{l}0 \\
1 \\
2\end{array}$ & $\begin{array}{l}2.001 \pm 0.001 \\
1.996 \pm 0.001 \\
1.992 \pm 0.001\end{array}$ & 0.028 & 0.353 & -0.852 \\
\hline & EVI & $\begin{array}{l}0 \\
1 \\
2\end{array}$ & $\begin{array}{l}2.001 \pm 0.001 \\
1.997 \pm 0.001 \\
1.994 \pm 0.001\end{array}$ & 0.022 & 0.288 & -0.859 \\
\hline
\end{tabular}

8 bits, observing that the latter shows more complexity than Landsat.

The MF-spectra parameters from Landsat-7 are shown in Table 3. In this section we will compare the MF spectra and the vegetation indexes of the Ikonos-2 8 bits (Table 2). The $\alpha$ (1) values from the four bands of Landsat-7 are higher than the ones presented by Ikonos- 28 bits, indicating a more uniform space distribution. Comparing between the bands, there are not significant differences, contrary to the trend we observed among them in Ikonos-2 8 bits. The $\alpha$ (2) shows the same behaviour.

The amplitudes calculated $\left(\Delta^{+}\right.$and $\left.\Delta^{-}\right)$in Landsat-7 bands present few variations (Table 3 ). The amplitude $\Delta^{+}$ decreases from ETM+\#1 to ETM+\#3 and then presents an increase in ETM+\#4 (NIR) whereas the other amplitude $\Delta^{-}$ remains practically constant. Observing these parameters in Ikonos-2 8-bit bands (Table 2), there are variations in value and behaviour for the four bands. In this case, both $\Delta^{+}$and $\Delta^{-}$increase as the wavelength increases for the three visible bands, but decrease for the NIR band (IK\#4).

The AIs estimated on these MFS amplitudes on each Landsat-7 bands are positive, except for ETM+\#3 (red band). For the green band (ETM+\#3) the symmetry of the MFS is complete. The band that shows certain asymmetry is the blue band (ETM+\#1).
Regarding the vegetation indexes, estimated on Landsat-7 bands, we found the following. The bi-log plot of the partition function $(\chi(q, \delta))$ versus $\delta$ is plotted in Fig. 9 for both vegetation indexes. Each graph contains 9 points corresponding to the bands from which they were estimated. The linear fit was done with the same methodology as that for the four bands. EVI and NDVI show the same behaviour, and the same range of scale was selected achieving 8 pixels, as shown by arrows in Fig. 9.

The MF spectra $f(\alpha)$ of EVI and NDVI, estimated based on the Landsat-7 image, are shown in Fig. 8. Both vegetation indexes show differences mainly in the right side of the MFS (for $q$ negative values). Comparing the range of $f(\alpha)$ values in the vegetation indexes to the range obtained in the four bands (left column in Fig. 8), there is a remarkable contrast. Meanwhile the NIR band of 8 bits achieves $f(\alpha)$ value close to 1.6, EVI and NDVI achieve values closer to 1. A similar situation was found with both images of Ikonos- 2 .

We are going to study the parameters obtained from the MF spectra for the vegetation indexes (Table 3 ). The results are quite similar to those found for the Landsat-7 bands, showing even higher values: 1.996 in NDVI and 1.997 in EVI.

The amplitude $\Delta^{+}$is quite low compared with the bands and to the vegetation indexes of Ikonos- 28 bits. On the other hand, the amplitude $\Delta^{-}$is higher than Landsat- 7 bands but 

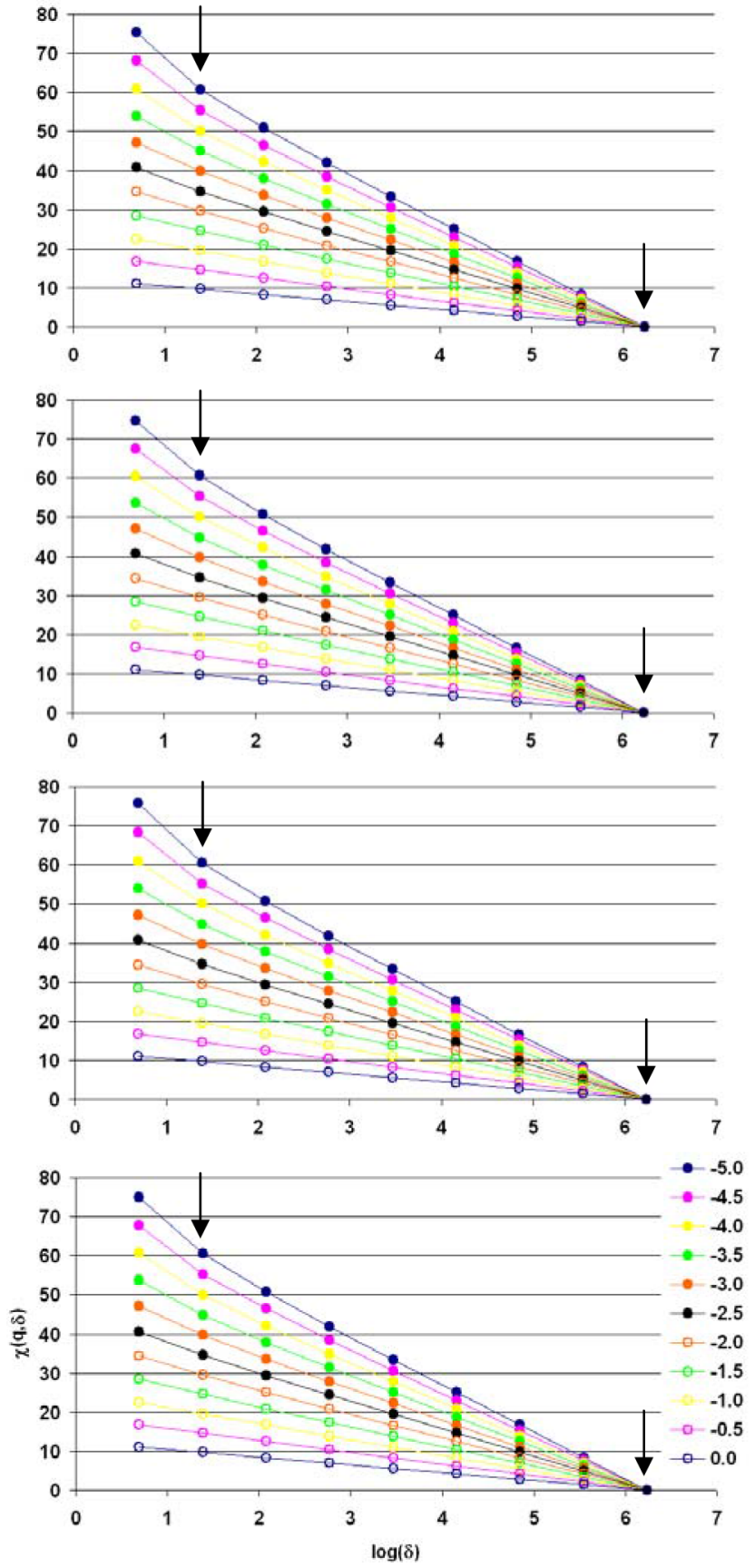

Figure 7. Bi-log plots of the partition function $\chi(q, \delta)$ versus $\delta$ for the first four bands of the Landsat-7 satellite and for $q<0$ values. From top to bottom we show the results for ETM+\#1, ETM+\#2, ETM+\#3 and ETM+\#4. The arrows marked the range of scales used for the fit and to calculate the slope for different values of $q$ ( 8 points).

by only a third of the values shown by Ikonos-2 8-bit vegetation indexes (Table 2). The AI estimated for both vegetation indexes are negative, indicating a high asymmetry on the right part of the MFS, as shown in Fig. 8. Comparing the AI values of Landsat-7 vegetation indexes with the ones of both Ikonos-2 images, these are the highest, indicating that the most unbalanced MFS shifted totally on the right side of the spectrum.

\section{Conclusions}

In this work, we have used MF spectra as a successful technique for analysing common information contained in multispectral images of the site of the Earth surface acquired by two satellites, Landsat-7 and Ikonos, in four common bands in the visible (blue, green and red) and near-infrared wavelength regions used in several vegetation indexes.

The radiometric resolution has been studied by comparing MF spectra of the images acquired by Ikonos- 2 coded in 11 bits and transformed into 8-bit code. The results obtained after the histogram transformation in the blue and green bands were those one would expect after the simplification applied from 11 to 8 bits, i.e. higher frequency in all the histogram bin values (see Fig. 2). In contrast, red and infrared bands showed no sensitivity at all to this transformation, keeping similar MF spectra. To our knowledge, this is the first time these differences among bands have been reported.

In order to analyse the effect of spatial resolution in each band at $4 \mathrm{~m}$ (Ikonos-2 with 8 bits) pixel size and $30 \mathrm{~m}$ (Landsat-7 with 8 bits) pixel size are compared. Obviously, the higher the spatial resolution, the higher the Hölder spectrum amplitudes in the green and blue bands are. In fact, observing the graphics of the three cases studied (Ikonos211 bits, Ikonos-2 8 bits and Landsat-7 8 bits) both bands gradually reduce their $\alpha(q)$ amplitude in the negative as well as in the positive $q$ values. However, this is not the case for red and NIR bands that present a much higher difference between Ikonos- 28 bits and Landsat- 7 curves of the MF spectra than between Ikonos- 211 and 8 bits.

In the $q>0$ MFS region for blue and green bands the sensitivity to both factors is very similar, the blue band ratio being slightly higher. The other two bands, red and NIR, for the same region, mainly present sensitivity to spatial resolution, showing a similar rate to blue and green bands. Observing the $q<0$ region for blue and green, the behaviour is similar to the positive one but with a lower ratio (between 1 and 2) and, once more, the red and infrared bands show slight sensitivity to radiometric resolution. Nevertheless, in the spatial resolution the red band has a ratio similar to blue and green, and NIR shows the highest ratio $(\sim 8)$, showing the extreme influence of the lowest values contained; see histograms in Fig. 2 (Ikonos-2 8 and 11 bits) and Fig. 6 (Landsat-7).

The implications of these variations in the blue, red and NIR in the multi-scaling behaviour of two vegetation indexes, NDVI and EVI, have been also studied. The radiometric resolution showed a higher influence in the MFS of the NDVI than in EVI. This implies that the use of the blue band in the latter has a steady effect on the scaling behaviour. 

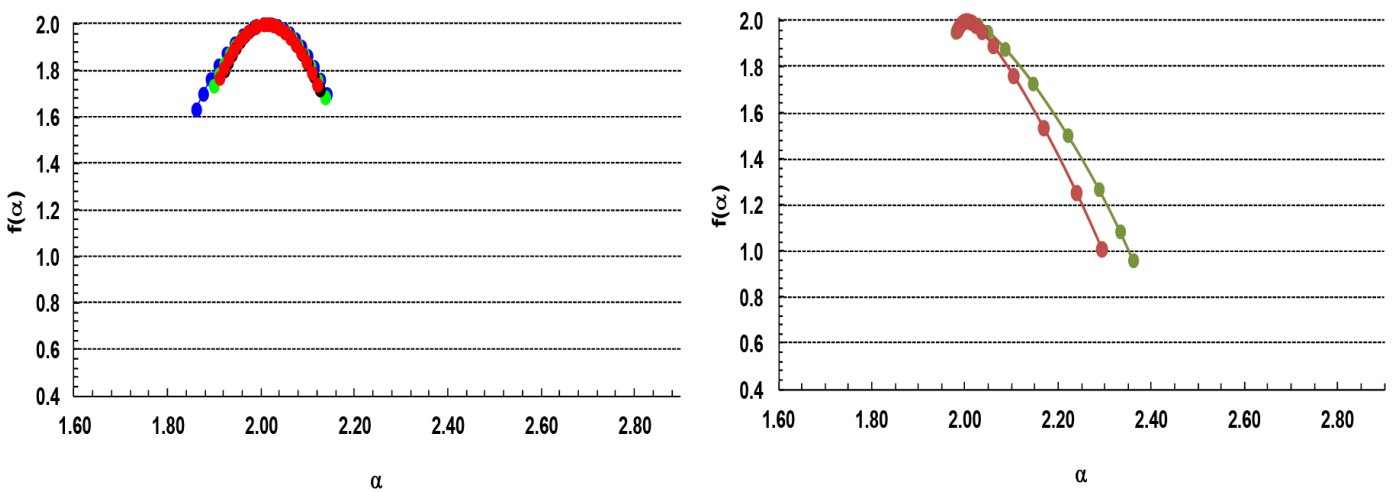

Figure 8. Multi-fractal spectrum of the Landsat-7 image for the original pixel values coded in 8 bits. Left plot corresponds to each band analysed: ETM+\#1 in blue colour, ETM+\#2 in green colour, ETM+\#3 in red colour and ETM+\#4 in black. Right plot corresponds to vegetation indexes: NDVI in green colour and EVI in brown.
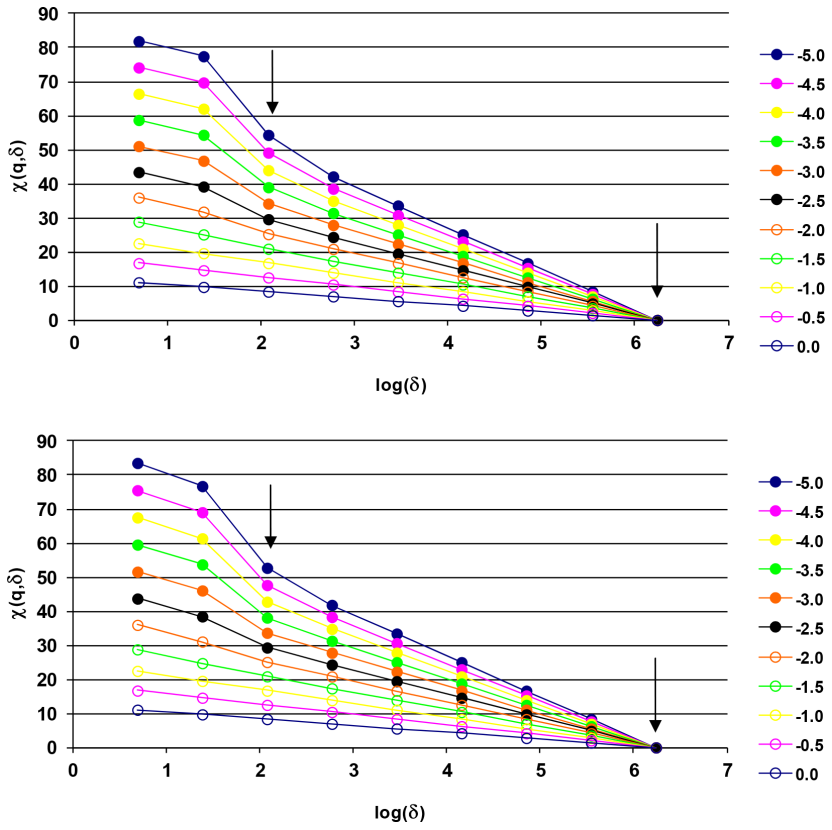

Figure 9. Bi-log plots of the partition function $\chi(q, \delta)$ versus $\delta$ for the vegetation indexes estimated from blue, red and NIR bands of the Landsat-7 satellite and for $q<0$ values. From top to bottom we show the results for NDVI and EVI. The arrows marked the range of scales used for the fit and to calculate the slope for different values of $q$ (7 points).

As was noted for the bands, the spatial resolution had a major impact in both vegetation indexes.

Further research will be conducted to establish a qualitative and quantitative comparison of these conclusions among several multi-fractal methodologies applied on these images.
Data availability. Data are available by email request to the corresponding author.

Competing interests. The authors declare that they have no conflict of interest.

Acknowledgements. Thanks are due to the anonymous referees and the editor for their interest in and patience with this work. Discussion and comments suggested by Jose Manuel Redondo are highly appreciated. This work has been supported by the Ministerio de Economía y Competitividad (MINECO) under contract nos. MTM2012-39101 and MTM2015-63914-P.

Edited by: Asim Biswas

Reviewed by: two anonymous referees

\section{References}

Aguado, P. L., Del Monte, J. P., Moratiel, R., and Tarquis, A. M.: Spatial Characterization of Landscapes through Multifractal Analysis of DEM, The Scientific World Journal, 2014, 563038, doi:10.1155/2014/563038, 2014.

Andraud, C., Beghdadi, A., and Lafait, J.: Entropic analysis of morphologies, Physica A, 207, 208-212, 1994.

Beaulieu, A. and Gaonac'h, H.: Scaling of differentially eroded surfaces in the drainage network of the Ethiopian plateau, Remote Sens. Environ., 82, 111-122, 2002.

Ben-Ze'ev, E., Karnieli, A., Agam, N., Kaufman, Y., and Holben, B.: Assessing Vegetation Condition In The Presence Of Biomass Burning Smoke By Applying The Aerosol-Free Vegetation Index (AFRI) On MODIS Images, Int. J. Remote Sens., 27, 3203-3221, 2006.

Carlson, T. N. and Ripley, D. A.: On the relation between NDVI, Fractional Vegetation Cover, and Leaf Area Index, Remote Sens. Environ., 62, 241-252, 1997. 
Cheng, Q.: A new model for quantifying anisotropic scale invariance and for decomposition of mixing patterns, Math. Geol., 36, 345-360, 2004.

Cheng, Q. and Agterberg, F. P.: Multifractal modelling and spatial statistics, Mathematical Geology, 28, 1-16, 1996.

De Cola, L.: Fractal analysis of a classified Landsat-7 scene, Photogrammetric Engineering and Remote Sensing, 55, 601-610, 1989.

Deng, F. P., Su, G. L., and Liu, C.: Seasonal Variation Of MODIS Vegetation Indexes And Their Statistical Relationship With Climate Over The Subtropic Evergreen Forest In Zhejiang, China, IEEE Geosci. Remote Sens. Lett., 4, 236-240, 2007.

Dial, G., Bowen, H., Gerlach, F., Grodecki, J., and Oleszczuk, R.: IKONOS satellite, imagery and products, Remote Sens. Environ., 88, 23-36, 2003.

Du, G. and Yeo, T. S.: A novel multifractal estimation method and its application to remote image segmentation, IEEE T. Geosci. Remote, 40, 980-982, 2002.

Evertsz, C. J. G. and Mandelbrot, B. B.: Chaos and Fractals: New Frontiers of Science, edited by: Peitgen, H., Jurgens, H., and Saupe, D., Springer-Verlag, New York, 921, 1992.

Feder, J.: Fractals, Plenum Press, New York, 283 pp., 1988.

Fioravanti, S., Multifractals: theory and application to image texture recognition, in: Fractals in Geosciences and Remote Sensing, Proceedings of a joint JRC/EARSeL Expert meeting, Ispra, Italy, 14-15 April 1994.

Gagnon, J. S., Lovejoy, S., and Schertzer, D.: Multifractal surfaces and terrestrial topography, Europhys. Lett., 62, 801-807, 2003.

Gaonac'h, H., Lovejoy, S., and Schertzer, D.: Resolution dependence of infrared imagery of active thermal features at Kilauea volcano, Int. J. Remote Sens., 24, 2323-2324, 2003.

Gouyet, J. F.: Physics and fractal structures, Springer-Verlag: New York, 1996.

Halsey, T. C., Jensen, M. H., Kadanoff, L. P., Procaccia, I., and Shraiman, B. I.: Fractal measures and their singularities: the characterization of strange sets, Phys. Rev. A, 33, 1141-1151, 1986.

Harvey, D. C., Gaonac'h, H., Lovejoy, S., and Sxhertzer, D.: Multrifractal characterization of remotely sensed volcanic features: a case study from Kilauea volcano, Hawaii, Fractals, 10, 265-274, 2002.

Hirata, T. and Imoto, M.: Multifractal analysis of spatial distribution of micro-earthquakes in the Kanto region, Geophys. J. Int., 107, 155-162, 1991 .

Huete, A., Miura, T., Yoshioka, H., Ratana, P., and Broich, M.: Indices of Vegetation Activity, in: Biophysical Applications of Satellite Remote Sensing, edited by: Hanes, J. M., Springer, 2014.

Kravchenko, A. N., Boast, C. W., and Bullock, D. G.: Multifractal analysis of soil spatial variability, Agron. J., 91, 1033-1041, 1999.

Laferrière, A. and Gaonac'h, H.: Multifractal properties of visible reflectance fields from basaltic volcanoes, J. Geophys. Res., 104, 5115-5126, 1999.

Lam, N. S.: Description and measurement of Landsat-7 TM images using fractals, Photogrammetric Engineering and Remote Sensing, 56, 187-195, 1990.

Lam, N. S. and De Cola, L.: Fractals in Geography, Prentice Hall, Englewood Cliffs, New Jersey, 1993.
Lavallée, D., Schertzer, D., and Lovejoy, S.: On the determination of the codimension function, in: Non-Linear Variability in Geophysics, edited by: Schertzer, D. and Lovejoy, S., Springer Netherlands: Dordrecht, the Netherlands, 99-109, 1991.

Lovejoy, S., Pecknold, S., and Schertzer, D.: Stratified multifractal magnetization and surface geomagnetic fields-I. Spectral analysis and modelling, Geophys. J. Int., 145, 112-126, 2001 a.

Lovejoy, S., Schertzer, D, Tessier, Y., and Gaonach, H.: Multifractals and resolution-independent remote sensing algorithms: the example of ocean colour, Int. J. Remote Sensing, 22, 119-1234, $2001 \mathrm{~b}$.

Lovejoy, S., Tarquis, A., Gaonac'h, H., and Schertzer, D.: Single and multiscale remote sensing techniques, multifractals and MODIS derived vegetation and soil moisture, Vadose Zone J., 7, 533-546, doi:10.2136/vzj2007.0173, 2008.

Maître, H. and Pinciroli, M.: Fractal characterization of a hydrological basin using SAR satellite images, IEEE Trans. Geosci. Remote, 37, 175-181, 1999.

Mandelbrot, B. B.: The Fractal Geometry of Nature, Freeman, San Francisco, 1983.

Hu, M.-G., Wang, J.-F., and Ge, Y.: Super-Resolution Reconstruction of Remote Sensing Images Using Multifractal Analysis, Sensors (Basel), 9), 8669-8683, doi:10.3390/s91108669, 2009.

Mika, A. M.: Three decades of Landsat-7 instruments, Photogrammetric Engineering and Remote Sensing, 63, 839-852, 1997.

Morató, M. C., Castellanos, M. T., Bird, N. R., and Tarquis, A. M.: Multifractal analysis in soil properties: Spatial signal versus mass distribution, Geoderma, 287, 54-65, doi:10.1016/j.geoderma.2016.08.004, 2017.

Parrinello, T. and Vaughan, R. A.: Multifractal Analysis and feature extraction in satellite imagery. Int. J. Remote Sens., 23, 1799$1825,2002$.

Qiu, H., Lam, N. S., Quattrochi, D. A., and Gamon, J. A.: Fractal characterization of hyperspectral imagery, Photogrammetric Engineering and Remote Sensing, 65, 63-71, 1999.

Renosh, P. R., Schmitt, F. G., and Loisel, H.: Scaling analysis of ocean surface turbulent heterogeneities from satellite remote sensing: use of 2D structure functions, PLoS ONE, 10, e0126975, doi:10.1371/journal.pone.0126975, 2015.

Sánchez, A., Serna, R., Catalina, F., and Afonso, C. N.: Multifractal patterns formed by laser irradiation in GeAl thin multilayer films, Phys. Rev. B, 46, 487-490, 1992.

Schertzer, D. and Lovejoy, S.: Physical modeling and analysis of rain and clouds by anisotropic scaling multiplicative processes, J. Geophys. Res., 92, 9693-9714, 1987.

Schmitt, F., Schertzer, D., Lovejoy, S., and Marchal, P.: Multifractal analysis of satellite images: towards an automatic segmentation, in: Fractals in Engineering, Arcachon: Jules, 103-109, 1997.

Tarquis, A. M., Losada, J. C., Benito, R., and Borondo, F.: Multifractal analysis of the Tori destruction in a molecular Hamiltonian System, Phys. Rev. E., 65, 0126213, doi:10.1103/PhysRevE.65.016213, 2001.

Tarquis, A. M., Giménez, D., Saa, A., Díaz, M. C., and Gascó, J. M.: Scaling and multiscaling of soil pore systems determined by image analysis, in: Scaling methods in soil physics, edited by: Pachepsky, Y., Radcliffe, D. E., and Magdi Selim, H., CRC Press, 2003.

Tarquis, A. M., Platonov, A., Matulka, A., Grau, J., Sekula, E., Diez, M., and Redondo, J. M.: Application of multifractal analysis to 
the study of SAR features and oil spills on the ocean surface, Nonlin. Processes Geophys., 21, 439-450, doi:10.5194/npg-21439-2014, 2014.

Tessier, Y., Lovejoy, S., Schertzer, D., Lavalle'e, D., and Kerman, B.: Universal multifractal indices for the ocean surface at far red wavelengths, Geophys. Res. Lett., 20, 1167-1170, 1993.

Turiel, A., Isern-Fontanet, J., García-Ladona, E., and Font, J.: Multifractal Method for the Instantaneous Evaluation of the Stream Function in Geophysics Flows, Phys. Rev. Lett., 95, 104502, doi:10.1103/PhysRevLett.95.104502, 2005.
Wang, X., Xie, H., Guan, H., and Xiaobing, Z.: Different responses of MODIS-derived NDVI to root-zone soil moisture in semi-arid and humid regions, J. Hydrol., 340, 12-24, 2007.

Xie, S., Cheng, Q., Xing, X., Bao, Z., and Chen, Z.: Geochemical multifractal distribution patterns in sediments from ordered streams, Geoderma, 160, 36-46, 2010. 